Revista PSICOLOGIA, 2017, Vol. 31 (1), 17-26. doi: 10.17575/rpsicol.v31i1.1140

\title{
Fratria: Tratamento parental diferenciado e estados emocionais negativos
}

\author{
Ana Filipa Pinheiro ${ }^{1}$, Otília Monteiro Fernandes ${ }^{1}$ \& Inês Carvalho Relva ${ }^{2}$ \\ ${ }^{1}$ Universidade de Trás-os-Montes e Alto Douro \\ 2 Universidade de Trás-os-Montes e Alto Douro; Centro de Estudos Sociais da Universidade de Coimbra
}

\begin{abstract}
Resumo: Para compreendermos a dinâmica familiar, temos de conhecer a constituição da família, pois são as características dos seus membros e das relações entre eles que influenciam essa dinâmica, nomeadamente, o número de filhos e as experiências diferenciadas, como a perceção da justiça e as doenças, que cada um deles tiveram e têm. Nesta amostra de 244 adolescentes verificámos que os adolescentes do sexo masculino sentem uma maior diferenciação ao nível do tratamento parental comparativamente aos adolescentes do sexo feminino, e que a depressão se correlaciona positivamente com o tratamento materno diferenciado, tendo este, assim, um contributo significativo para o aparecimento da depressão nos adolescentes. Como o tratamento materno diferenciado parece influenciar o bem-estar emocional dos adolescentes, parece-nos importante que se façam mais investigações de modo a compreender melhor qual o seu impacto e as suas repercussões na vida dos filhos, quer na adolescência, quer na infância.
\end{abstract}

Palavras-chave: Tratamento parental diferenciado; Relação entre irmãos; Estados emocionais; Relação parental.

Sibling: Parental differential treatment and negative emotional states: To understand the family dynamics, first we have to know the structure of the family, as some of its features may influence the relationship between its members, as the perception of justice and disease that each had and have. In this sample of 244 adolescents, the results demonstrated no differences in the emotional well-being in adolescents. However, it is concluded that adolescent males feel a greater differentiation in terms of parental treatment compared to female adolescents. It was established that depression is positively correlated with differentiated maternal treatment, thus having a significant contribution to the onset of depression in adolescents. Since the differentiated maternal treatment appears to influence the emotional well-being of adolescents, it seems relevant to continue the research in this area of studies in order to understand the real impact on the lives of children, either in adolescence or in childhood.

Keywords: Parental differential treatment; Siblings relationship; Emotional states; Parental relationship.

A base para a descoberta do mundo exterior ocorre na família, porque esta é o primeiro meio para a integração do indivíduo e para o seu desenvolvimento psicossocial. Uma parte de nós e da nossa personalidade é construída através das interações com os nossos familiares, especialmente, pais e irmãos (quando os há), uma vez que são eles que convivem connosco nos primeiros anos de vida (Fernandes, Alarcão, \& Raposo, 2007).

A relação fraterna é considerada a relação mais extensa de todo o ciclo vital do ser humano, desempenhando um papel fundamental na dinâmica familiar (Meynckens-Fourez, 1999), pois é com os irmãos que estabelecemos as nossas primeiras experiências com os pares, moldando assim a nossa maneira de ser, pensar e agir (Fernandes, 2002). Contudo, sabemos que as relações fraternas são muito complexas e são caraterizadas por um misto de sentimentos: se por um lado temos o companheirismo, a solidariedade e a entreajuda (Goldsmid \& Féres-Carneiro, 2011), por outro, temos o ciúme, a rivalidade e a violência (Relva, Fernandes, \& Alarcão, 2012). Muitos fatores podem contribuir para uma boa ou má relação entre os irmãos, e um deles, que tem suscitado um grande interesse na sociedade científica, é o tratamento parental diferenciado, isto é, o modo diferenciado como cada um dos pais trata cada um dos filhos. Vários têm sido, aliás, os autores que têm encontrado uma relação próxima entre a negatividade das relações fraternas e o tratamento parental diferenciado (e.g., Brody, Stoneman, \& McCoy, 1992; Dunn \& Kendrick, 1981a, 1981b).

${ }^{1}$ Endereço para correspondência: Universidade de Trás-os-Montes e Alto Douro, Departamento de Educação e Psicologia, Pólo 1 da ECHS, Quinta de Prados, 5000-801 Vila Real E-mail: tila@utad.pt 
Quais os fatores que levam os pais a tratarem de forma diferente os filhos? De que forma o tratamento diferenciado influencia o estado emocional dos filhos? Muitos são os fatores que influenciam o tratamento parental diferenciado e a relação entre os irmãos desde a ordem de nascimento (Ng, Mofrad, \& Uba, 2014), o estado emocional das crianças (Feinberg, Neiderhiser, Simmens, Reiss, \& Hetherington, 2000), a perceção de justiça quanto ao tratamento diferenciado (Kowal, Kramer, Krull, \& Crick, 2002), a personalidade dos pais (Browne, Meunier, O'Connor, \& Jenkins, 2012) e a deficiência (Cuzzocrea, Larcan, \& Costa, 2014).

O tratamento parental diferenciado é um fenómeno complexo e inevitável, sendo que para o compreender é necessário termos em consideração determinados aspetos (Dunn \& Kendrick, 1981a, 1981b, 1986; Furman \& Buhrmester, 1985a) como, por exemplo: o meio familiar não partilhado (Daniels \& Plomin, 1985); o nascimento de cada um dos filhos - uma vez que na sua maioria ocorrem em diferentes épocas e circunstâncias da vida, e consequentemente, as condições emocionais e materiais dos pais serão diferentes, por exemplo (Barroso, 2008) -, mas também relativamente aos vários comportamentos que cada género deverá ter e assumir na vida familiar.

Kowal et al. (2002) estudaram a perceção de justiça, ao nível do tratamento parental diferenciado, em adolescentes entre os 11 e os 17 anos de idade. Os resultados encontrados indicam que existem duas formas de tratamento em que os filhos podem percecionar índices de maior diferenciação parental: o facto de sentirem que são desfavorecidos injustamente, ou então, que são favorecidos injustamente. E aquilo que influencia o bem-estar emocional dos filhos não é o facto de serem favorecidos ou não, mas sim a forma como percecionam a justiça do tratamento parental, ou seja, o fundamental é que os filhos sintam que recebem o que realmente merecem e necessitam. Os autores enunciam, ainda, que a equidade do tratamento parental está relacionada com respostas emocionais positivas, como uma maior autoestima e um reduzido número de problemas de comportamento de internalização.

Também, Boll, Ferring, e Filipp (2005) investigaram os efeitos do tratamento parental diferenciado na qualidade da relação entre os irmãos e os pais. Verificaram que a relação entre os irmãos é melhor quando o tratamento parental é tido como justo, mencionando ainda, por um lado, que quando os pais são idosos, o irmão que se considerou desfavorecido não apresenta tanto empenho nos cuidados com os mesmos, enquanto o irmão favorecido sentirá uma maior responsabilidade e uma obrigação para ajudar os pais.

Já Ripoll, Carrillo e Castro (2008) investigaram os sistemas relacionais entre pais e filhos, e a sua influência no ajuste psicológico nas díades de irmãos adolescentes. Verificaram que quando os adolescentes percecionam uma relação positiva com os pais, os níveis de comunicação e afeto com os irmãos são mais positivos, assim como o seu ajustamento psicológico.

$\mathrm{Ng}$ et al. (2014) investigaram o efeito da ordem do nascimento no tratamento parental diferenciado em adolescentes asiáticos, entre os 13 e os 17 anos de idade. Apuraram que ao nível da afetividade parental, não se obtiveram resultados significativos, contudo, ao nível do controlo, verificaram-se algumas diferenças: os mais velhos relatam um maior controlo parental relativamente aos mais novos. Segundo os autores, uma possível explicação, para estes resultados, tem a ver com o nível de maturidade das crianças, pois, algumas vezes, as crianças mais velhas tendem a ser "substitutas" dos pais. Já Poonam e Punia (2012) avaliaram o impacto dos fatores parentais e contextuais no tratamento diferenciado dos filhos ao nível do afeto, privilégios e disciplina. Concluíram que os pais e as mães eram mais carinhosos com os irmãos mais novos das díades do mesmo sexo. Contudo, em díades do sexo oposto, as mães eram mais afetuosas com os rapazes, quer fossem o mais velho ou o mais novo, enquanto os pais eram relativamente mais carinhosos com as meninas, independentemente da ordem de nascimento.

Scholte, Engels, Kemp, Harackch e Ovrerbeek (2007) procuraram perceber o impacto que o tratamento parental diferenciado e a relação entre os irmãos têm na delinquência, durante a infância. Os autores verificaram que a ordem do nascimento é um fator importante, na medida em que os irmãos mais novos são mais vulneráveis aos efeitos do tratamento parental diferenciado do que os irmãos mais velhos. Apuraram, ainda, que quando os rapazes sentiam que eram desfavorecidos, o seu comportamento estava relacionado com o vandalismo e a delinquência, enquanto que nas raparigas, o ato de roubar era o mais explícito. Verificaram também que nas díades do mesmo sexo, os irmãos mais novos parecem ser os mais influenciados pelo tratamento materno diferenciado; e relativamente ao facto dos irmãos mais velhos serem menos vulneráveis, os autores consideram dever-se a razões como a maior responsabilidade e maturidade destes.

A adolescência é caracterizada por uma fase de transição/mudança, onde o organismo humano sofre grandes desenvolvimentos ao nível físico, mas também psicológico e social, de modo a adaptar-se às exigências da sociedade (Eisenstein, 2005). Sabemos que a adolescência é um período importante do ciclo vital, e a comparência de problemas emocionais como a ansiedade, depressão e stresse poderão ser um 
obstáculo para o bom desenvolvimento biopsicossocial do indivíduo.

Os problemas emocionais, assim como a depressão, são problemas de saúde que têm vindo a crescer a nível mundial, entre as crianças e os adolescentes, sendo as perturbações de ansiedade e de humor as mais frequentes nesta população (Shavers, 2014).

Bhasin, Sharma e Saini (2010) estudaram a ansiedade, depressão e stresse (ADS), em adolescentes com idades compreendidas entre os 13 e os 18 anos de idade de famílias afluentes, bem como os possíveis fatores que contribuiriam para níveis mais elevados de ADS. Os autores verificaram que nesta faixa etária os jovens apresentavam valores elevados de ansiedade, depressão e stresse, salientando que ao nível da depressão o sexo feminino sobressaía em relação ao sexo oposto. Encontraram ainda uma ligação entre o nível de escolaridade e os níveis de ADS nos jovens, o que levou a concluir que a pressão para um bom desempenho académico tem influência no estado emocional do indivíduo, uma vez que nos jovens com menor rendimento escolar os níveis de ADS eram mais elevados.

Já Siddiqui e Rehman (2014) preocuparam-se em compreender os níveis de ansiedade académica em relação ao sexo e estatuto socioeconómico em alunos do ensino secundário. Verificaram que a ansiedade académica é um problema presente nos alunos do ensino secundário, todavia averigua-se que são os alunos do sexo feminino que apresentam valores mais elevados comparativamente com o sexo masculino. E salientaram ainda que o estatuto socioeconómico é um fator importante na compreensão desta problemática, uma vez que afeta negativamente as pessoas com um nível socioeconómico mais baixo.

Como é mencionado por Kowal, Krull e Kramer (2004), é inevitável que o tratamento dos pais seja diferente para cada um dos filhos, uma vez que cada um tem as suas próprias especificidades e necessidades; todavia salienta-se que embora este fenómeno seja ineludível, o modo como os filhos percecionam a forma como são tratados define até que ponto esta diferenciação poderá ser favorável ou desfavorável para o seu desenvolvimento biopsicossocial, pois desde que os filhos vejam as suas necessidades satisfeitas, poderão avaliar positivamente as suas relações com os pais e com os irmãos.

Em suma, o tratamento diferenciado dos pais será definido pela forma como os adolescentes o entendem e o aceitam, o que irá moldar os seus comportamentos e atitudes, influenciando assim a sua relação com os irmãos, com os pais e o seu estado psicológico, não esquecendo que cada um tem as suas particularidades e os seus próprios recursos para lidar com as adversidades. Em Portugal, são ainda poucos os estudos que abordam o tratamento parental diferenciado e as suas consequências para os filhos, embora seja um tema pertinente para a psicologia do desenvolvimento individual e familiar. Assim, o presente trabalho procura desenvolver uma visão mais aprofundada desta temática e tem como objetivo geral compreender de que forma o tratamento parental diferenciado poderá influenciar o bemestar (estados emocionais) nos filhos adolescentes. Colocamos, então, como objetivos principais do presente estudo: (a) determinar a percentagem de adolescentes que consideram existir semelhanças/diferenças ao nível do tratamento parental; (b) estudar em que medida o tratamento parental diferenciado varia em função do sexo e idade dos adolescentes; (c) verificar as associações entre os sintomas emocionais negativos e o tratamento parental diferenciado; e, por último, (d) analisar se o tratamento parental diferenciado é um possível preditor dos sintomas emocionais negativos.

\section{MÉTODO}

\section{Participantes}

A amostra foi constituída por 244 adolescentes com irmãos, com idades compreendidas entre os 12 e 18 anos $(M=15.01 ; D P=1.86)$ tendo mais de metade $(58.6 \%)$ do sexo feminino. Os participantes frequentavam o 3. o ciclo de escolaridade e ensino secundário, sendo que 46 (18.9\%) se encontravam no 7.․ ano, 28 (11.5\%) no 8o ano, 41 (16.8\%) no 9.o ano. Relativamente ao ensino secundário, 47 (19.3\%) estudavam no 10. ano, 45 (18.4\%) no 11.․a ano e 37 (15.2\%) frequentavam o 12. ano. No que respeita ao número de irmãos, a maioria (75.8\%) tinha somente um irmão, 16\% tinha dois irmãos, 3.7\% tinha três irmãos, $4.1 \%$ tinha quatro irmãos e $4 \%$ cinco irmãos.

\section{Instrumentos}

O Questionário Sociobiográfico (QSB) é um questionário adaptado do Social Environment Questionnaire (SEQ) de Toman (1993), por Fernandes e Relva (2013), e questiona o sujeito sobre si próprio (sexo, idade, naturalidade, ano de escolaridade, doenças), assim como a sua posição na fratria (número de irmãos, tipo, sexo, idade, doença ou deficiência) e situação atual dos progenitores (idade, ano de escolaridade e estado civil).

O Sibling Inventory Differential Experience (SIDE) de Daniels e Plomin (1985), versão traduzida por Fernandes e Relva (2012), permite-nos avaliar as experiências dos irmãos ao nível da interação fraterna, 
tratamento diferenciado pelos pais (mãe e pai, separadamente), caraterísticas dos pares e dos eventos específicos individuais. Uma vez que este instrumento questiona o participante apenas relativamente a um irmão, quando o participante tem mais do que um irmão é-lhe solicitado que responda em relação ao que lhe é mais próximo em idade. Cada item apresenta-nos cinco opções de resposta (1-"mais vezes com o meu irmão/irmã"; 2-"ligeiramente mais vezes com ele/ela"; 3-"igual"; 4-"ligeiramente mais vezes comigo"; 5-"muitas mais vezes comigo"). Tendo como base o objetivo do presente estudo, apenas foi utilizada a escala referente ao tratamento parental diferenciado, que nos dá 4 índices ou subescalas (controlo e afeto materno; controlo e afeto paterno), por exemplo: "o meu pai/mãe punha-nos na ordem ou disciplinava-nos" (escala controlo) ou "o meu pai/mãe ficava orgulhoso (a) das coisas que fazíamos" (escala afeto) (Daniels \& Plomin, 1985).

Analisadas individualmente, os valores de confiabilidade para as 4 escalas do tratamento parental diferenciado, foram de .67 para o afeto materno; .58 para o controlo materno; .86 para o afeto paterno e .72 para o controlo paterno. Realizou-se uma análise factorial confirmatória de primeira ordem, tendo sido verificados valores adequados dos índices de ajustamento $\chi^{2}(28)=639.163 ; p=0.03$; Ratio $=22.83$; $\mathrm{CFI}=.98 ; \mathrm{SRMR}=.03$ e RMSEA $=.06$.

A Depression Anxiety Stress Scale (DASS) de Lovibond e Lovibond (1995), versão portuguesa (EADS-C-21) adaptada por Leal, Antunes, Passos, Pais-Ribeiro e Maroco (2009), permite-nos avaliar características e comportamentos associados aos níveis de ansiedade, depressão e stresse. 0 formato das respostas está estruturado segundo uma escala tipo Likert com 4 possibilidades de resposta (0-"não se aplicou nada a mim"; 1-“aplicou-se a mim algumas vezes"; 2-“aplicou-se a mim muitas vezes; 3-"aplicou-se a mim a maior parte das vezes"). Contém itens para avaliar cada uma das três dimensões abrangidas pelo instrumento, sendo que o stresse é avaliado pelos itens $1,6,8,11,12,14$, 18; a depressão pelos itens: 3 , 5, $10,13,16,17$ e 21 e a ansiedade pelos itens $2,4,7,9,15,19$ e 20 . No que concerne a consistência interna, o valor da escala geral para a presente amostra é adequado $(\alpha=.94)$. Relativamente às subescalas, os valores de confiabilidade, para a depressão foram de $\alpha=.88$; na ansiedade foi obtido um valor de $\alpha=.85$; e para o stresse $\alpha=.84$. No que diz respeito à análise factorial confirmatória, esta revelou valores dos índices de ajustamento adequados, sendo $\chi^{2}(36)=1285.903 ; p=.000 ;$ Ratio $=35.72 ;$ CFI $=.97 ;$ SRMR $=$ .04 e RMSEA $=.08$.

\section{Procedimentos}

Realizou-se o contacto com os agrupamentos de escolas de forma a obtermos o consentimento dos diretores das mesmas. De seguida, enviámos os protocolos e os pedidos de autorização para os responsáveis pelo jovem, através dos diretores de turma. Depois da autorização dada pelos encarregados de educação procedeu-se à entrega dos protocolos aos jovens, para preenchimento na sala de aula, tendo sido explicado os objetivos gerais da investigação, proporcionando os dados essenciais para o preenchimento dos questionários e evidenciando-se a participação voluntária, bem como a confidencialidade e o anonimato das respostas. Depois de preenchidos os questionários em sala de aula, os participantes entregaram-nos ao professor responsável de cada turma, tendo sido posteriormente recolhidos pela investigadora.

\section{Estratégia de Análise de Dados}

Para o estudo dos dados recolhidos, foi efetuada uma análise dos instrumentos e criada uma base de dados com o auxílio do programa estatístico Statistical Package for the Social Sciences - IBM SPSS, versão 21; e para a execução das propriedades psicométricas dos respetivos instrumentos, utilizou-se o programa Structural Equation Modeling Software - EQS for Windows, versão 6.1. Criaram-se as dimensões referentes a cada um dos instrumentos, efetuando-se as devidas análises psicométricas ao nível da consistência interna com recurso ao alpha de Cronbach e dos estudos fatoriais confirmatórios. No que concerne a análise de dados, estimou-se a frequência do tratamento parental diferenciado; realizaram-se análises de variância multivariada (MANOVAS), com nível de significância de $5 \%$ (p $\leq .05)$; o teste $t$ para a comparação de médias em amostras independentes; executaram-se correlações de Pearson determinando-se a força da relação entre as variáveis de acordo com as linhas de interpretação de Cohen (1988); e, por fim, procedeu-se à realização de uma regressão múltipla hierárquica, na qual houve a necessidade de codificar a variável sexo como variável dummy, sendo concedido o valor zero ao sexo feminino e o valor um ao sexo masculino. 


\section{RESULTADOS}

\section{Análise Descritiva: Médias e Desvio-Padrão das Dimensões do SIDE}

$\mathrm{Na}$ Tabela 1 podem observar-se os valores absolutos e relativos referentes à dimensão tratamento parental diferenciado. A média dos valores absolutos $(0$ = não existem diferenças na experiência dos irmãos; 1 = alguma diferença na experiência dos irmãos; 2 = muita diferença na experiência dos irmãos) é de .28 para o tratamento parental diferenciado.

Tabela 1. Médias e Desvio-Padrão das dimensões do SIDE

\begin{tabular}{|c|c|c|}
\hline Dimensões do SIDE & $\begin{array}{l}\text { Valores Relativos } \\
\text { (Média } \pm \text { DP) }\end{array}$ & $\begin{array}{c}\text { Valores Absolutos } \\
\text { (Média } \pm \text { DP) }\end{array}$ \\
\hline Afeto Diferenciado Materno & $2.90 \pm .38$ & $.24 \pm .37$ \\
\hline Controlo Diferenciado Materno & $3.12 \pm .47$ & $.35 \pm .42$ \\
\hline Afeto Diferenciado Paterno & $2.96 \pm .46$ & $.23 \pm .42$ \\
\hline Controlo Diferenciado Paterno & $3.09 \pm .47$ & $.28 \pm .44$ \\
\hline
\end{tabular}

Nota. SIDE=Sibling Inventory Differential Experience

Procurou-se assim determinar qual é a percentagem de irmãos que classificam a perceção do tratamento parental diferenciado de modo semelhante ou díspar. Verificou-se que $80.8 \%$ consideram não existirem diferenças ao nível do afeto materno e $81.6 \%$ para o afeto paterno; $12.2 \%$ e $13.5 \%$ consideram existirem algumas diferenças (materno e paterno respetivamente) enquanto $4.9 \%$ e $4.8 \%$ reportam a existência de muitas diferenças ao nível do afeto, materno e paterno, respetivamente. Relativamente ao nível do controlo, 73.1\% consideram não haver diferenças ao nível do controlo materno e $78 \%$ ao nível do controlo paterno; $18.3 \%$ e $15.5 \%$ referem a presença de algumas diferenças (materno e paterno, respetivamente), e por último, $8.5 \%$ e $6.4 \%$ consideram a existência de muitas diferenças ao nível do controlo parental (materno e paterno, respetivamente).

\section{Análise Diferencial das Dimensões do SIDE em Função do Sexo}

Para comparar os valores relativos do tratamento parental diferenciado (ao nível do afeto e controlo) em função do sexo, efetuou-se uma análise mediante o test $t$, para amostras independentes. Os resultados obtidos (Tabela 2) demonstraram a presença de diferenças estatisticamente significativas em função do sexo ao nível do tratamento parental diferenciado nas dimensões afeto materno $[t(169.849)=-2.991 ; p=$ .003 ], com IC 95\% [-.24,-.05], controlo materno [ $t$ (185.483) $=-3.011 ; p=.003]$, com IC 95\% [-.33,-.07], afeto paterno $[t(167.037)=-3.822 ; p=.000]$, com IC 95\% [-.32,-.10] e controlo paterno $[t(164.781)=-$ $4.314 ; p=.000]$, com IC 95\% [-.45,-.17], sendo o sexo masculino a percecionar um tratamento mais diferenciado ao nível do afeto e controlo materno $(M=.33 ; D P=.42, M=.56 ; D P=.56$, respetivamente), bem como ao nível do afeto e controlo paterno $(M=.39 ; D P=.47$ e $M=.54 ; D P=.62$, respetivamente) comparativamente com o sexo feminino, onde foram obtidos os seguintes valores ao nível do afeto e controlo materno $(M=.19 ; D P=.30, M=.36 ; D P=.45$, respetivamente $)$ e ao nível afeto e controlo paterno $(M=.18 ; D P=.33, M=.23 ; D P=.42$, respetivamente).

Tabela 2. Diferença das dimensões do SIDE em função do sexo

\begin{tabular}{|c|c|c|c|c|}
\hline SIDE & Sexo & $M \pm D P$ & IC $95 \%$ & $\begin{array}{c}\text { Direção das diferenças } \\
\text { significativas }\end{array}$ \\
\hline \multirow{2}{*}{ Afeto diferenciado materno } & 1-Feminino & $.19 \pm .30$ & \multirow{2}{*}[-.24,-.05]{} & \multirow{2}{*}{$2>1$} \\
\hline & 2-Masculino & $.33 \pm .42$ & & \\
\hline \multirow{2}{*}{ Controlo diferenciado materno } & 1-Feminino & $.36 \pm .45$ & \multirow{2}{*}[-.33,-.07]{} & \multirow{2}{*}{$2>1$} \\
\hline & 2-Masculino & $.56 \pm .56$ & & \\
\hline \multirow{2}{*}{ Afeto diferenciado paterno } & 1-Feminino & $.18 \pm .33$ & \multirow{2}{*}[-.32,-.10]{} & \multirow{2}{*}{$2>1$} \\
\hline & 2-Masculino & $.39 \pm .47$ & & \\
\hline \multirow{2}{*}{ Controlo diferenciado paterno } & 1-Feminino & $.23 \pm .42$ & \multirow{2}{*}[-.45,-.17]{} & \multirow{2}{*}{$2>1$} \\
\hline & 2-Masculino & $.54 \pm .62$ & & \\
\hline
\end{tabular}

Nota. SIDE=Sibling Inventory Differential Experience

Análise Diferencial das Dimensões do SIDE em Função do Sexo da Idade

Visando investigar a presença de diferenças do tratamento parental diferenciado em função da idade realizou-se uma análise de variância multivariada (MANOVA). Os resultados observados (Tabela 3) demonstraram que ao nível do tratamento materno diferenciado existem diferenças estatisticamente significativas em função da idade $\left[\mathrm{F}(2,241)=3.786 ; p=.02 ; \eta^{2}=.03\right.$; Wilks $\left.\lambda=.970\right]$, sendo o grupo de jovens entre os 16 e os18 anos de idade que demonstram uma maior perceção da diferenciação do 
tratamento ao nível do afeto $(M=.31 ; D P=.38)$ e controlo $(M=.53 ; D P=.57)$ materno, comparativamente com os jovens entre os $12-15$ anos ao nível do afeto $(M=.20 ; D P=.33)$ e controlo $(M=$ .38; $D P=.45$ ) materno.

Tabela 3. Diferença das dimensões do SIDE em função da idade

\begin{tabular}{|c|c|c|c|c|}
\hline SIDE & Grupos de idades & $\mathrm{M} \pm \mathrm{DP}$ & IC $95 \%$ & $\begin{array}{c}\text { Direção das diferenças } \\
\text { significativas }\end{array}$ \\
\hline \multirow{2}{*}{ Afeto diferenciado materno } & $1-12-15$ & $.20 \pm .33$ & {$[.15, .26]$} & \multirow{2}{*}{$2>1$} \\
\hline & $2-16-18$ & $.31 \pm .38$ & {$[.24, .38]$} & \\
\hline \multirow{2}{*}{ Controlo diferenciado materno } & $1-12-15$ & $.38 \pm .45$ & {$[.30, .46]$} & \multirow{2}{*}{$2>1$} \\
\hline & $2-16-18$ & $.53 \pm .57$ & {$[.43, .63]$} & \\
\hline \multirow{2}{*}{ Afeto diferenciado paterno } & $1-12-15$ & $.22 \pm .40$ & {$[.16, .29]$} & \multirow{2}{*}{ n.s. } \\
\hline & $2-16-18$ & $.32 \pm .41$ & {$[.24, .40]$} & \\
\hline \multirow{2}{*}{ Controlo diferenciado paterno } & $1-12-15$ & $.31 \pm .48$ & {$[.23, .40]$} & \multirow{2}{*}{ n.s. } \\
\hline & $2-16-18$ & $.42 \pm .59$ & {$[.32, .53]$} & \\
\hline
\end{tabular}

Nota. SIDE=Sibling Inventory Differential Experience

\section{Análise Inferencial: Associação entre o Tratamento Parental Diferenciado e os Sintomas} Emocionais Negativos

Quando correlacionadas as dimensões do tratamento parental diferenciado e dos sintomas emocionais negativos (Tabela 4), verificaram-se associações positivas, mas de magnitude baixa, entre a dimensão afeto diferenciado materno e a depressão $(r=.150 ; p \leq .05)$, bem como nas dimensões controlo diferenciado materno e a depressão ( $r=.126 ; p \leq .05)$.

Tabela 4. Associações entre variáveis do EADS-C-21 e SIDE, média e desvio padrão (N=244)

\begin{tabular}{lcccccccc}
\hline & $\mathbf{1}$ & $\mathbf{2}$ & $\mathbf{3}$ & $\mathbf{4}$ & $\mathbf{5}$ & $\mathbf{6}$ & $\mathbf{7}$ & $\boldsymbol{M} \pm \boldsymbol{D P}$ \\
\hline 1. Afeto Diferenciado Materno & - & - & - & - & - & - & - & $.24 \pm .37$ \\
2. Controlo Diferenciado Materno & $.534^{* *}$ & - & - & - & - & - & - & $.35 \pm .42$ \\
3. Afeto Diferenciado Paterno & $.450^{* *}$ & $.370^{* *}$ & - & - & - & - & - & $.23 \pm .42$ \\
4. Controlo Diferenciado Paterno & $.352^{* *}$ & $.494^{* *}$ & $.677^{* *}$ & - & - & - & - & $.28 \pm .44$ \\
5. Depressão & $.150^{*}$ & $.126^{*}$ & .023 & .019 & - & - & - & $6.58 \pm 5.52$ \\
6. Ansiedade & .082 & .103 & .001 & .047 & $.733^{* *}$ & - & - & $4.71 \pm 4.72$ \\
7. Stresse & .094 & .077 & .008 & .039 & $.762^{* *}$ & $.770^{* *}$ & - & $7.18 \pm 5.00$ \\
\hline
\end{tabular}

\section{Análise Preditiva: Predição da Depressão em Função do Sexo e do Tratamento Parental Diferenciado}

De modo a analisar quais as variáveis independentes que melhor predizem o aparecimento da depressão, realizaram-se análises de regressões múltiplas hierárquicas, sendo que, para cada dimensão desta, foram inseridos dois blocos. 0 bloco 1 corresponde à variável sexo (dummy); o bloco 2 ao tratamento materno diferenciado e ao tratamento paterno diferenciado, realizados separadamente (Tabela 5).

Tabela 5. Análise preditiva: Predição da Depressão

\begin{tabular}{|c|c|c|c|c|c|c|c|}
\hline Depressão & $R 2$ & R2 change & $B$ & $S E$ & $\beta$ & $p$ & $t$ \\
\hline $\begin{array}{l}\text { Bloco } 1 \\
\text { Sexo (Dummy) }\end{array}$ & .004 & .004 & -.733 & .717 & -.066 & .308 & -1.021 \\
\hline \multicolumn{8}{|l|}{ Bloco 2} \\
\hline Mãe & .033 & .029 & & & & & \\
\hline Afeto D. Materno & & & 2.217 & 1.111 & .144 & .047 & 1.996 \\
\hline Controlo D. Materno & & & .573 & .785 & .053 & .466 & .730 \\
\hline Pai & .005 & .001 & & & & & \\
\hline Afeto D. Paterno & & & .265 & 1.063 & .020 & .803 & .249 \\
\hline Controlo D. Paterno & & & .138 & .822 & .013 & .867 & .168 \\
\hline
\end{tabular}

No que concerne à depressão, verifica-se que apenas o bloco 2 referente ao tratamento materno diferenciado teve um contributo significativo $[\mathrm{F}(3,81)=2.740 ; p=.044]$ e explica $3.3 \%$ da variância do modelo $\left(\mathrm{R}^{2}=.033\right)$, contribuindo individualmente com $2.9 \%$ da variância para o modelo $\left(\mathrm{R}^{2}\right.$ change = .029); sendo que o bloco 1 não apresentou contributos significativos $[\mathrm{F}(1,31)=1.043 ; p=.308]$. Neste sentido, e analisando individualmente o contributo de cada uma das variáveis independentes dos blocos, 
verifica-se que uma das variáveis apresenta uma contribuição significativa ( $\mathrm{p} \leq .05)$, afeto mãe $(\beta=.144)$ enquanto variável preditora da depressão. Verificando-se que o tratamento paterno diferenciado não apresenta valores significativos para esta variável (bloco $1[\mathrm{~F} 1,31)=1.043 ; p=.308]$; bloco $2[\mathrm{~F} 3,12)=$ $.408 ; p=.748])$.

\section{DISCUSSÃO}

O tratamento parental diferenciado é inevitável, pois cada um dos filhos tem as suas próprias caraterísticas e individualidades (Kowal et al., 2004). Desde sempre existiram diferenças na forma como os pais se relacionam com os filhos, uma vez que existem variáveis que condicionam o seu próprio relacionamento, como a idade, o sexo, a posição na fratria, a maior ou menor semelhança física/psíquica com um familiar amado/odiado, entre outros. 0 modo como os filhos (primeiramente crianças e, depois, adolescentes) percecionam este meio influenciará, certamente, a sua forma de agir, pensar e sentir física e emocionalmente.

No que diz respeito à percentagem de adolescentes que consideram um tratamento parental diferenciado similar ou diferente, verificamos neste estudo que existe uma variabilidade no relacionamento com os pais, sendo que grande parte dos adolescentes percecionam o tratamento parental de modo semelhante em relação a todos os filhos, mas uma pequena parte dos participantes refere diferenças no modo como os pais os tratam a si mesmos e ao(s) seu(s) irmão(s), indo de encontro ao que é mencionado pelos primeiros investigadores deste tema, Daniels e Plomin (1985). 0 que nos leva assim a colocar algumas hipóteses, nomeadamente: 1) o facto de a maioria dos participantes considerarem que o tratamento recebido pelos pais é igualitário talvez se deva à fase de desenvolvimento dos participantes por nós estudados, pois provavelmente ainda estão incapazes de criticar os pais, por estarem ainda a desenvolver o processo de construção da sua identidade, isto é, ainda não estão totalmente separados dos pais; 2) adicionalmente, não sabemos o tipo de relacionamento com o irmão a que se refere o participante, sendo que se tratando- do irmão "mais próximo em idade", o tratamento parental poderá ser visto como mais igualitário; 3) finalmente, pode-se equacionar a pressão da desejabilidade social, tal como sugerido por Coldwell, Pike e Dunn (2008).

No que concerne às diferenças do tratamento parental em função do sexo, os resultados apontam no sentido de os jovens do sexo masculino sentirem mais diferenças ao nível do tratamento parental comparativamente com os jovens do sexo feminino. Alguns autores (e.g., Bègue \& Roché; 2005; Poonam \& Punia, 2012) sugerem que o sexo feminino tende a ser mais protegido pelos pais. Assim sendo, espera-se que as jovens avaliem o tratamento parental como sendo adequado e que satisfaz as suas necessidades, como temos vindo a ver em estudos anteriormente realizados (Boll et al., 2005; Feinberg et al., 2000 É assim que está nas referências). 0 mais importante é que os mesmos sintam que recebem o que necessitam, isto é, que o tratamento que recebem esteja de acordo com as suas especificidades (Faber \& Mazlish, 1995; Feinberg et al., 2000).

Em relação à faixa etária, registaram-se diferenças significativas, sendo que são os irmãos que se enquadram entre os 16 -18 anos de idade que referem maior diferenciação ao nível do tratamento parental. Alguns estudos ( $\mathrm{Ng}$ et al., 2014) referem que são os mais velhos que indicavam mais diferenciação ao nível do tratamento dado pelos pais, nomeadamente ao nível do controlo paternal, não sendo visíveis diferenças nas restantes dimensões. Os autores consideram que possivelmente isso se deve ao facto de os mais velhos serem mais responsáveis e com mais maturidade, o que lhes permite uma perceção mais objetiva das diferenças existentes entre os irmãos e a correspondente diferença no comportamento parental em relação a cada um dos filhos, o que também foi encontrado e enunciado por Poonam e Punia (2012). Nós colocamos assim a hipótese de que os jovens, com o avançar da idade, desenvolvem uma melhor apreciação relativamente ao tratamento parental. E talvez essa avaliação crítica face aos comportamentos parentais seja necessária e uma consequência decorrente do processo de separação que os adolescentes mais velhos estão a fazer relativamente aos pais. Quanto mais velhos, mais os adolescentes estão separados/individualizados e, consequentemente, mais realisticamente olham para os pais idealizados da infância.

Relativamente às associações entre as dimensões do tratamento parental diferenciado e dos sintomas emocionais negativos encontramos uma associação positiva significativa, ainda que baixa, entre a depressão e o tratamento materno diferenciado (nomeadamente, o afeto e o controlo). De acordo com vários autores (Fernandes, 2002; Hoskins, 2014), o comportamento parental influência de forma diferente os adolescentes e o modo como os próprios lidam com os acontecimentos/adversidades da vida quotidiana, e o comportamento e características das mães parecem ser mais influenciadoras dos comportamentos dos filhos. Por exemplo, num estudo realizado por Cardoso, Rodrigues e Vilar (2004), verificou-se que existiam diferenças quanto à presença de sintomas depressivos nos adolescentes quando relacionados com a profissão e a escolaridade da mãe, isto é, quando a escolaridade era mais baixa ou 
quando trabalhava em casa os adolescentes demonstravam mais sintomas depressivos, mas o mesmo não foi verificado relativamente aos pais.

Procurou-se ainda verificar qual o efeito preditor do tratamento parental diferenciado e os sintomas emocionais negativos. Os resultados evidenciaram o efeito preditor do tratamento materno diferenciado, em particular o afeto materno, no aparecimento da depressão nos adolescentes. De acordo com Skinner, Johnson e Synder (2005), a relação entre pais e filhos é estabelecida através de diversas variáveis, o que de certo modo influencia a forma como os adolescentes se desenvolvem ao nível biopsicossocial. 0 presente resultado demonstra-nos que somos influenciados pelo modo como nos relacionamos com os nossos progenitores, e especialmente como o afeto materno modela o nosso desenvolvimento. 0 que também é provado no estudo de Reeves e Bamaca (2012), que verificou que a intensidade dos conflitos entre as mães e as adolescentes mexicanas é um forte preditor no desenvolvimento de sintomas depressivos, enquanto a frequência dos conflitos entre mães e filhas é um forte preditor nos problemas das relações românticas. Também Finkelstein, Donenberg e Martinovich (2001) procuraram perceber a relação entre o controlo materno e a depressão na adolescência, mas tendo em conta, como variável moderadora, a etnia das participantes da amostra em estudo. Os autores concluíram que altos níveis de controlo materno estavam associados a menos sintomas depressivos no caso das adolescentes africanas, já na amostra caucasiana foi encontrado o oposto, i.e. quanto maior o controlo, maior o desenvolvimento de sintomas depressivos nas jovens. Verificaram, também, que as adolescentes de etnia africana reportaram níveis mais elevados de controlo, concluindo assim que a etnia é um fator importante a ter em atenção na relação entre pais e filhos. Em relação ao efeito preditor da depressão no tratamento parental diferenciado, apesar de não ter sido analisado no presente estudo, poderá também ocorrer, como aliás foi verificado por Brody, Stoneman e McCoy (1992). Estes autores verificaram que as pequenas diferenças entre os irmãos em termos de emotividade negativa podem, com o tempo, fazer aparecer altos níveis de tratamento diferenciado por parte dos pais, o que pode, por sua vez, aumentar as diferenças de níveis de emotividade negativa entre os membros da fratria. Também Daniels, Dunn, Furstenberg e Plomin (1985), num estudo conduzido junto de 348 famílias com dois filhos adolescentes com idades compreendidas entre os 11 e os 17 anos, verificaram que irmãos mais ajustados psicologicamente também experienciavam mais proximidade materna. Neste estudo também foi verificado que os adolescentes que tinham recebido mais expectativas parentais positivas eram, também, os que apresentavam maiores índices de ajustamento, demonstrando que também o comportamento parental quando é (e é percecionado como sendo) desigual parece contribuir para o desenvolvimento diferenciado dos irmãos (cf. Fernandes, 2002).

No que respeita às implicações práticas, desejamos que este estudo possa ser um impulsionador para pesquisas mais aprofundadas sobre os efeitos do tratamento parental diferenciado no bem-estar emocional dos filhos, uma vez que encontramos associações positivas entre as duas variáveis, bem como compreender realmente quais as variáveis e influências que poderão estar envolvidas na forma como os filhos classificam e percecionam o tratamento parental diferenciado. No que diz respeito à elevada percentagem de participantes que consideraram referir não existir diferenças ao nível do tratamento parental, será necessário analisar estes resultados à luz da faixa etária dos participantes, sendo que a diferentes idades poderão corresponder diferentes perceções, pelo que os estudos longitudinais seriam de extrema importância para explorar este aspeto. Seria pertinente também estudar mais do que uma criança por família, uma vez que os estudos intrafamiliares nos dariam uma melhor e maior dimensão das reais diferenças no tratamento parental. E, em suma, temos de ter presente, como Deal, Halverson e Wampler (1994) observaram, que determinados irmãos são muito semelhantes em termos de temperamento, comportamento e tratamento parental, embora outros sejam menos similares.

Para finalizar, mencionamos algumas limitações do presente estudo que devem ser tidas em consideração. A primeira limitação remete-nos para o facto de os instrumentos serem de autorrelato e nós, enquanto investigadores, não poderemos perceber se realmente aquilo que os jovens assinalam corresponde ao que sentem e pensam ou ao que se passa realmente. Aliás, esta limitação, i.e. o facto de se tratarem de perceções subjetivas dos participantes, foi inicialmente salientada por Daniels e Plomins (1985). Esta limitação poderia ser colmatada cruzando informações obtidas através de questionários aos pais e restante fratria, ou, ainda melhor, fazendo observações objetivas, tal como sugerido pelos autores anteriormente referidos. Assim, seria importante obtermos a perceção de todos os irmãos relativamente ao tratamento parental, uma vez que cada um apresenta a sua própria perceção, bem como perceber de que modo estas perceções influenciam as relações fraternas, parentais e, até, conjugais.

Tendo como ponto de partida os nossos resultados e as conclusões de estudos anteriormente realizados, verificamos que o modo como se desenvolve a dinâmica familiar influencia o bem-estar emocional dos jovens, todavia, existe a necessidade de investigações mais aprofundadas e que explorem melhor a contribuição do tratamento parental diferenciado nas mais diversificadas variáveis da vida dos 
filhos. Como vimos ao longo de toda a investigação, o tratamento parental diferenciado sempre foi um fenómeno presente nas famílias. Torna-se, assim, fundamental realizar mais investigações neste âmbito, a fim de compreender e aprofundar o verdadeiro impacto do comportamento parental percebido no bemestar emocional dos filhos adolescentes, e explorar o modo como os jovens interpretam esta relação, tendo em conta o sexo e a idade. Seria também importante aprofundar a relação existente entre a dinâmica familiar e o surgimento de sintomas depressivos, e de como intervir junto das famílias para auxiliar na diminuição da prevalência deste problema de saúde mental, tão comum atualmente.

\section{Referências}

Barroso, M. (2008). Fratrias e género: Contributos para uma análise sociológica das relações fraternas. Comunicação apresentada do VI Congresso Português de Sociologia: Mundos Sociais Saberes e Práticas. Faculdade de Ciências Sociais e Humanas, Lisboa.

Bègue, L., \& Roché, S. (2005). Birth order and youth delinquent behaviour testing the differential parental control hypothesis in a French representative sample. Psychology, Crime \& Law, 11(1), 73-85. https://doi.org/10.1080/1068316042000221121.

Bhasin, S., Sharma, R., \& Saini, N. (2010). Depression, anxiety and stress among adolescent students belonging to affluent families: A school-based study. Indian Journal of Pediatrics, 77, 161-165. https://doi.org/10.1007/s12098-009-0260-5.

Boll, T., Ferring, D., \& Filipp, S. (2005). Effects of parental differential treatment on relationship quality with siblings and parents: Justice evaluations as mediators. Social Justice Research, 18(2), 155-182. https://doi.org/10.1007/s11211-005-7367-2.

Brody, G. H., Stoneman, Z., \& McCoy, J. K. (1992). Associations of maternal and paternal direct and differential behaviour with sibling relationships: Contemporaneous and longitudinal analyses. Child Development, 63, 82-92. https://doi.org/10.1111/j.1467-8624.1992.tb03597.x

Browne, D., Meunier, J., O'Connor, T., \& Jenkins, J. (2012). The role of parental personality traits in differential parenting. Journal of Family Psychology, 26(4), 542-553. https://doi.org/10.1037/a0028515.

Cardoso, P., Rodrigues, C., \& Vilar, A. (2004). Prevalência de sintomas depressivos em adolescentes portugueses. Análise Psicológica, 22(4), 667-675. https://doi.org/10.14417/ap.264.

Cohen, J. (1988). Statistical power analysis for the behavioral sciences. Hillsdale, NJ: Lawrence Erlbaum Associates.

Coldwell, J., Pike, A., \& Dunn, J. (2008). Maternal differential treatment and child adjustment: A multiinformant approach. Social Development, 17(3), 596-612. https://doi.org/10.1111/j.14679507.2007.00440.x.

Cuzzocrea, F., Larcan, R., \& Costa, S. (2014). Parent's competence and social skills in siblings of disabled children. Social Behavior and Personality, 42(1), 45-58. https://doi.org/10.2224/sbp.2014.42.1.45.

Daniels, D., \& Plomin, R. (1985). Differential experience of siblings in the same family. Developmental Psychology, 21(5), 747-760. https://doi.org/10.1037/0012-1649.21.5.747.

Daniels, D., Dunn, J., Furstenberg, F. F., \& Plomin, R. (1985). Environmental differences within the family and adjustment differences within pairs of adolescent siblings. Child Development, 56, 764-774. https://doi.org/10.2307/1129765.

Deal, J. E., Halverson, C. F., \& Wampler, K. S. (1994). Sibling similarity as an individual differences variable: Within family measures of shared environment. In E.M. Hetherington, D. Reiss, \& R. Plomin, Separate social worlds of siblings: The impact of nonshared environment on development (pp. 205218). Hillsdale, NJ: Lawrence Erlbaum Associates.

Dunn, J., \& Kendrick, C. (1986). Hermanos y hermanos. Madrid: Alianza Editorial.

Dunn, J., \& Kendrick, C. (1981a). Interaction between young sibling: Association with the interaction between mother and firstborn child. Development Psychology, 17(3), 336-343. https://doi.org/10.1037/0012-1649.17.3.336.

Dunn, J., \& Kendrick, C. (1981b). Social behavior of young siblings in the same family context: differences between same-sex and different-sex dyads. Child Development, 52, 1265-1273. https://doi.org/10.2307/1129515.

Eisenstein, E. (2005). Adolescência: Definições, conceitos e critérios. Adolescência \& Saúde, 2(2), 6-7.

Faber, A., \& Mazlish, E. (1995). Jalousies et rivalités entre frères et soeurs. Paris: Éditions Stock.

Feinberg, M., Neiderhiser, J., Simmens, S., Reiss, S., \& Hetherington, M. (2000). Sibling comparison of differential parental treatment in adolescence: Gender, self-esteem, and emotionality as mediators of the parenting-adjustment association. Child Development, 71(6), 1611-1628. https://doi.org/10.1111/1467-8624.00252.

Fernandes, O. (2002). Semelhanças e diferenças entre irmãos. Lisboa: Climepsi. 
Fernandes, O. M., \& Relva, I. C. (2012). Sibling Inventory Differential Experience - SIDE. Manuscrito não publicado. Universidade de Trás-os-Montes e Alto Douro, Vila Real.

Fernandes, O. M., \& Relva, I. C. (2013). Social Environment Questionnaire (SEQ) . Manuscrito não publicado. Universidade de Trás-os-Montes e Alto Douro, Vila Real.

Fernandes, O., Alarcão, M., \& Raposo, J. (2007). Posição na fratria e personalidade. Estudos de Psicologia, 24(3), 297-304. http://dx.doi.org/10.1590/S0103-166X2007000300001.

Finkelstein, J., Donenberg, G., \& Martinovich, Z. (2001). Maternal control and adolescent depression: Ethnic differences among clinically referred girls. Journal of Youth and Adolescence, 30(2), 155-170. https://doi.org/10.1023/A:1010341724157.

Furman, W., \& Buhrmester, D. (1985a). Children's perceptions of the qualities of sibling relationships. Child Development, 56, 448-461. https://doi.org/10.2307/1129733

Goldsmid, R., \& Féres-Carneiro, T. (2011). Relação fraterna: Constituição do sujeito e formação do laço social. Psicologia USP, 22(4), 771-787. https://doi.org/10.1590/S0103-65642011005000031

Hoskins, D. (2014). Consequences of parenting on adolescent outcomes. Societies, 4, 506-531. https://doi.org/0.3390/soc4030506.

Kowal, A., Kramer, L., Krull, J., \& Crick, N. (2002). Children's perceptions of the fairness of parental preferential treatment and their socioemotional well-being. Journal of Family Psychology, 16(3), 297-306. https://doi.org/10.1037//0893-3200.16.3.297.

Kowal, A., Krull, J., \& Kramer, L. (2004). How the differential treatment of siblings is linked with parentchild relationship quality. Journal of Family Psychology, 18(4), 658-665. https://doi.org/10.1037/0893-3200.18.4.658.

Leal, I., Antunes, R., Passos, T., Pais-Ribeiro, J., \& Maroco, J. (2009). Estudo da Escala de Depressão, Ansiedade e Stresse para crianças (EADS-C). Psicologia, Saúde \& Doenças, 10(2), 277-284.

Lovibond, S., \& Lovibond, P. (1995). Manual for the Depression Anxiety Stress Scales (DASS). New South Wales: Psychology Foundation Monograph.

Meynckens-Fourez, M. (1999). La fratrie, le point de vue éco-systémique. In E. Tilmans-Ostyn \& M. Meynckens-Fourez (Eds.), Les ressources de la fratrie (pp. 37-68). Ramonville Saint-Agne: Éditions Érès.

Ng, W., Mofrad, S., \& Uba, I. (2014). Effect of birth order on the differential parental treatment of children. Asian Social Science, 10(14), 132-137. https://doi.org/10.5539/ass.v10n14p132.

Pooman, P., \& Punia, S. (2012). Impact of parental and contextual facts on differential treatment of siblings in the families. Studies on Home and Community Science, 6(2), 107-112.

Reeves, D., \& Bamaca, M. (2012). A longitudinal examination of parent-adolescent conflict, romantic relationship conflict, and depressive symptoms among mexican-origin adolescent females. Pennsylvania: Department of Human Development and Family Studies, University of Pennsylvania. $\begin{array}{llll}\text { Retrieved } & \text { Outubro } & 05, & 2015,\end{array}$ http://forms.gradsch.psu.edu/diversity/mcnair/mcnair_jrnl2012_14/files/2012-Reeves.pdf.

Relva, I., Fernandes, O. M., \& Alarcão, M. (2012). Violência entre irmãos: Uma realidade desconhecida. Revista Interamericana de Psicologia, 46(3), 375-384. https://doi.org/10.13140/RG.2.1.2801.8800.

Ripoll, K., Carrillo, S., \& Castro, J. (2008). Relación entre hermanos y ajuste psicológico en adolescentes: Los efectos de la calidad de la relación padres-hijos. Advances en Psicologia Latinoamericana, 27(1), 125-142.

Scholte, R., Engels, R., Kemp, R., Harakch, Z., \& Overbeek, G. (2007). Differential parental treatment, siblings' relationships and delinquency in adolescence. Journal Youth Adolescence, 36, 661-671. https://doi.org/10.1007/s10964-006-9155-1.

Shavers, C. (2014). Commentary: Emotional problems and depression among children and adolescent in today's society. Open Journal of Depression, 3, 74-87. https://doi.org/10.4236/ojd.2014.32012

Siddiqui, M., \& Rehman, A. (2014). An international study of academic anxiety in relation to socioeconomic status, gender and school type among secondary school students. International Journal of Education Research and Technology, 5(2), 74-79. https://doi.org/10.15515/ijert.09764089.5.2.7479

Skinner, E., Johnson, S., \& Snyder, T. (2005). Six dimensions of parenting: A motivational model. Parenting Science and Practice, 5(2), 175-232. https://doi.org/10.1207/s15327922par0502_3.

Toman, W. (1993). Family constellation: Its effects on personality and social behavior. New York: Springer Publishing Company. 